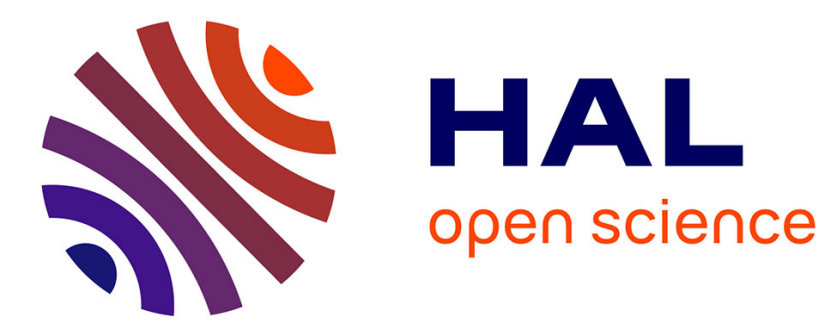

\title{
Transformateur à haut voltage. A survolteur cathodique P. Villard
}

\section{- To cite this version:}

P. Villard. Transformateur à haut voltage. A survolteur cathodique. J. Phys. Theor. Appl., 1901, 10 (1), pp.28-32. 10.1051/jphystap:019010010002801 . jpa-00240509

\section{HAL Id: jpa-00240509 https://hal.science/jpa-00240509}

Submitted on 1 Jan 1901

HAL is a multi-disciplinary open access archive for the deposit and dissemination of scientific research documents, whether they are published or not. The documents may come from teaching and research institutions in France or abroad, or from public or private research centers.
L'archive ouverte pluridisciplinaire HAL, est destinée au dépôt et à la diffusion de documents scientifiques de niveau recherche, publiés ou non, émanant des établissements d'enseignement et de recherche français ou étrangers, des laboratoires publics ou privés. 


\section{TRANSFORMATEUR A HAUT VOLTAGE}

A SUryolteur cathodique;

Par M. P. VILLARD.

J'ai décrit, il y a environ un an $\left({ }^{1}\right)$, une soupape électrique permettant de trier, ou de redresser, les courants alternatifs à haut voltage. A l'occasion de ces expériences, M. Carpentier a eu l'obligeance de construire et de mettre gracieusement à ma disposition un transformateur à l'aide duquel j'ai pu continuer l'étude de cette question.

(1) Comptes Rendus, t. CXXVIII, p. 994; 1899.

Dans une note des Beiblïtler (t. XXIII, p. 686; 1899) M. E. Wiedemann considère l'appareil en question comme une application des résultats publiés par M. A. Wehnelt (Wied. Ann., t. LXV, p. 510 ; p. 1898). Cette assertion ne me paraît pas justifiée. La soupape cathodique, représentée à part (fig. 1), est un perfectionnement des appareils analogues dont l'idée première remonte à Gaugain et à Riess, et dont llittorf a fait une étude détaillée dans son premier mémoire sur les rayons cathodiques (Pogg. Ann., t. CXXXVI, p. 1; 1869'. Cet auteur a parfaitement reconnu l'influence des dimensions des électrodes sur la résistance d'un tube à gaz raréfié et il a certainement entrevu le rôle des parois. C'est précisément l'étude de ces phénomènes, en particulier de la répulsion exercée par les parois sur ce que j'ai appelé l'afflux cathodique, qui m'a permis de réaliser sans tâtonnements un appareil beaucoup plus dissymétrique que celui de Hittorf, mais fonctionnant de la même manière, et à faire d'un objet de démonstration connu depuis trente ans un appareil de laboratoire.

La grande résistance présentée par la petite électrode quand elle est cathode tient à sa faible dimension et à l'étranglement placé immédialement devant elle pour réduire le débit de l'afflux, Ne fonctionnant que comme anode, cette électrode ne s'échauffe pas. L'électrode en spirale placée loin des parois et présentant un grand développement constitue au contraire une cathode à grand débit, par suite peu résistante, et elle peut évidemment s'échauffer sans inconvénient. 
Transformateur. - Le transformateur, à circuit magnétique fermé, consomme 7 à 8 ampères sous 110 volts ( 42 périodes) et donne entre les bornes secondaires 50.000 volts efficaces. Quand on amène lentement le courant primaire à sa valeur normale, la distance explosive secondaire, mesurée entre des boules de 2 centimètres de diamèlre, est de 9 centimètres. Elle peut atteindre accidentellement 20 centimètres, si on établit brusquement le courant primaire $\left(^{(1)}\right.$.

L'étincelle ainsi obtenue est une véritable flamme, de la grosseur du doigt; entraînée par l'air qu'elle échauffe, elle s'élève au-dessus de l'excitateur en formant un arc sinueux, dont le développement peut s'élever à 30 ou 40 centimètres. Elle est accompagnée d'un ronflement particulier qui tient à la périodicité de la décharge.

L'énergie mise en jeu dans ce phénomène est supérieure à ò kilowatts, et l'intensité primaire dépasse 60 ampères quand la décharge se produit.

On peut modérer ces effets en intercalant un rhéostat dans le circuit primaire, et on arrive à obtenir une étincelle semblable à celle de la bobine de Ruhmkorff, c'est-à-dire un trait de feu avec ou sans auréole.

Ce transformateur, muni ou non d'un appareil redresseur, pourrait à la rigueur être utilisé tel quel. Toutefois sa puissance en rendrait le maniement dangereux et l'usage assez difficile dans le laboratoire, où la diversité des expériences peut se résumer en disant que la source électrique doit pouvoir être mise en court circuit sans que l'intensité de la décharge prenne une valeur exagérée. D'autre part, le voltage indiqué plus haut serait, dans bien des cas, insuffisant, et, pour l'augmenter il faudrait accroître le poids déjà considérable de lappareil.

Les dispositifs suivants ont pour but de remédier à ces divers inconvénients.

Dispositif secondaire. - Les extrémités libres $\mathrm{SS}^{\prime} \mathrm{du}$ fil secondaire (fig. 1 moins la soupape) sont fixées à demeure aux armatures $\mathrm{AA}^{\prime}$ de deux condensateurs en micanite fixés dans la boîte qui renferme le transformateur. Les armatures $\mathrm{BB}^{\prime}$ sont munies de deux bornes $\mathrm{bb}^{\prime}$ auxquelles on altache les rhéophores conduisant par exemple à un excitateur $\mathrm{EE}^{\prime}$.

(1) 11 arrive souvent qu'à l'inslant du démarrage l'intensité prirnaire dans un transformateur dépasse momentanément sa valeur normale; de là un survoltage passager. 
Le circuit secondaire est ainsi coupé par les condensateurs, comme dans l'alternateur à haute fréquence de M. Tesla. La quantité d'électricité mise en jeu dans chaque décharge ne peut donc dépasser un maximum défini par la capacité des condensateurs, et fixé d'avance à une valeur compatible avec le bon fonctionnement du transformateur et des appareils récepteurs.

On obtient alors, entre les boules de l'excitateur, un flot d'étincelles brillantes qui ne présentent jamais l'aspect de flamme caractéristique des courants intenses. On peut, sans inconvénient, rapprocher jusqu'au contact les boules $\mathrm{EE}^{\prime}$, ou les relier par un appareil de résistance minime, et le rapprochement accidentel des rhéophores ne détermine plus une décharge intense, capable de décupler l'intensité du courant primaire. En même temps, tout danger pour l'opérateur a disparu.

On peut à volonté réduire la puissance des décharges et le débit maximum. Il suffit d'ajouter un ou deux petits condensateurs supplémentaires (condensateurs de machine statique, par exemple) en série avec les premiers. On est ainsi absolument maître de la puissance utilisée, et les conditions de chaque expérience sont faciles à définir exactement.

L'expérience a montré que la dépense d'énergie nécessaire pour obtenir des étincelles beaucoup plus fortes que celles d'une bobine de Ruhmkorff, est presque insignifiante. L'intensité primaire reste en effet à peu près la même que pendant la marche à vide. L'énergie consommée par le transformateur est peu supérieure à celle qui correspond à la perte par hystérésis.

Disons, en passant, que le dispositif précédent convient parfaitement pour obtenir l'étincelle condensée, servant, par exemple, à la production des courants à haute fréquence. Il suffit de relier l'excitaleur $\mathrm{EE}^{\prime}$ aux armatures internes d'un couple de bouteilles de Leyde.

Soupape survoltrice. - L'appareil qui vient d'être décrit est l'équivalent d'une machine statique très puissante, mais alternative. $\mathrm{Au}$ moyen des soupapes électriques on peut, soit redresser les courants comme je l'ai déjà indiqué, soit éliminer l'une des alternances, ce qui est ici préférable. Pour cela on dispose entre les bornes $b b^{\prime}$ ( $f g .1$ ) une soupape NP quin'offre qu'une cohésion diélectrique insignifiante quand l'électrode $\mathrm{N}$ est cathode, mais résiste à 60.000 volts pour l'alternance inverse. L'une des alternances passe donc par la soupape et l'autre par 
l'excitateur, ou par l'appareil qui le remplace, un tube de Crookes par exemple. La différence de potentiel en EE' passe ainsi alternativement par une valeur très faible (quelques milliers de volts) et une valeur très élevée, comme avec une bobine de Ruhmkorff. Ce léger inconvénient, d'ailleurs facile à supprimer, est amplement compensé par l'avantage suivant :

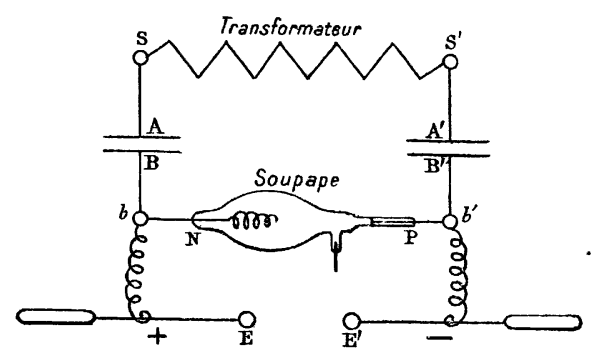

FIG. 1.

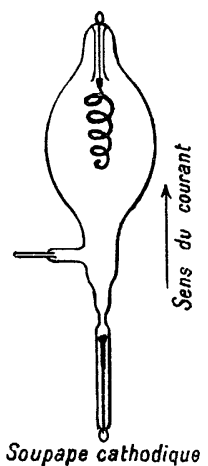

Soupape cathodique

Le rôle de la soupape NP ne consiste pas seulement à éliminer l'une des alternances ; le voltage disponible est en même temps considérablement accru. L'étincelle qu'on obtient entre les boules $\mathrm{EE}^{\prime}$ atteint 18 centimètres au lieu de 9 : entre pointes la distance explosive s'élève à 24 centimètres. Il ne m'est pas possible actuellement de donner de ce phénomène une explication suffisamment fondée.

Dans la pratique, en raison de ce voltage élevé, il convient d'employer non pas une, mais deux soupapes en série. La cohésion diélectrique de chacune d'elles ne dépasse guère en effet 60.000 volts, et il est évidemment préférable de laisser un peu d'élasticité à leur réglage.

L'entretien de ces soupapes est fort simple, il consiste simplement à y introduire un peu d'hydrogène en chauffant dans une flamme le tube de platine dont elles sont munies. L'utilité de cette opération, qui se fait en marche, se reconnaît à ce que les rayons cathodiques émanés de la cathode $\mathbf{N}$ deviennent assez énergiques pour provoquer la fluorescence du verre de l'ampoule.

Comme précédemment, des condensateurs additionnels peuvent être ajoutés en série avec ceux du transformateur, avant la soupape. 
Subdivision du courant secondaire. - Le transformateur-condensateur permet de résoudre un problème analogue à celui de la division de la lumière électrique. La puissance de l'appareil étant très supérieure à celle dont on a besoin, on peut sans inconvénient alimenter deux groupes de condensateurs disposés en parallèle comme le montre la fig. 2, et faire fonctionner deux appareils soit simultanément, c'est-à-dire sur la même alternance, soit sur des alternances opposées. Dans la fig. 2 les choses sont disposées de telle sorte que les étincelles $\mathrm{EE}^{\prime}, \mathrm{E}_{1} \mathrm{E}_{1}{ }_{1}$ soient alternantes. Il suffirait de retourner la soupape $\mathrm{N}_{1} \mathrm{P}_{1}$ pour qu'elles soient simultanées.

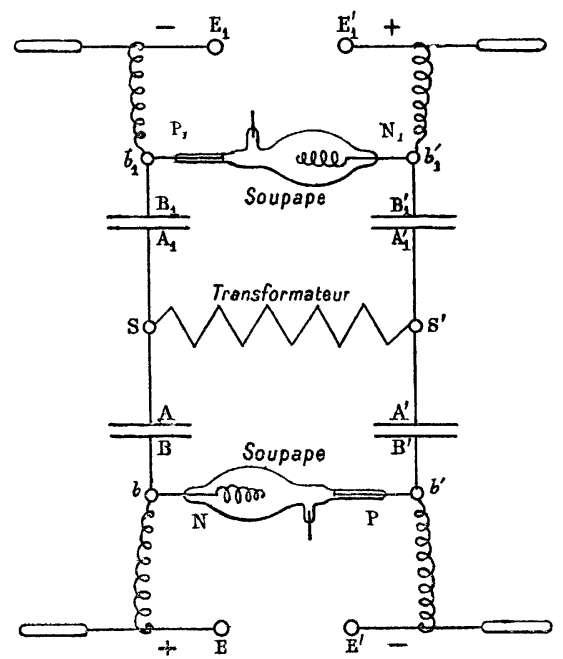

FIG. 2.

Les deux installations $\mathrm{AA}^{\prime} \mathrm{BB}^{\prime} \mathrm{NP}$ et $\mathrm{A}_{1} \mathrm{~A}_{1}^{\prime} \mathrm{B}_{1} \mathrm{~B}_{1}^{\prime} \mathrm{N}_{1} \mathrm{P}_{1}$ sont indépendantes et, par suite, il est inutile que les appareils placés en $\mathrm{EE}^{\prime}, \mathrm{E}_{\mathbf{1}} \mathrm{E}_{1}^{\prime}$, aient les mêmes constantes électriques. On peut par exemple mettre l'un des excitateurs en court circuit sans que l'autre cesse de fonctionner. En un mot on dispose de deux sources électriques périodiques d'égale puissance, mais distinctes. Rien n'empêche d'ailleurs de relier ces deux sources en quantité dans le cas où un seul circuit est utilisé : on double ainsi le débit disponible. 